Revista Interdisciplinaria de Humanidades, Educación, Ciencia y Tecnología

Año VII. Vol. VII. N¹3. Julio - Diciembre. 2021

Hecho el depósito de ley: pp201602FA4721

ISSN-L: 2542-3029; ISSN: 2610-802X

Universidad Nacional Experimental Francisco de Miranda (UNEFM). Santa Ana de Coro. Venezuela

Ruth Eleana Herrera-García; Ana María Vargas-Castillo; Mery Marleny Martínez-lbarra

Angela Beatriz Rodríguez-Montes

DOI $10.35381 / \mathrm{cm} . v 7 i 13.530$

\title{
Influencia del COVID-19 en el desempeño laboral de los docentes de
} Educación Básica

\section{Influence of COVID-19 on the job performance of Basic Education teachers}

\author{
Ruth Eleana Herrera-García \\ rutheleana.herrerag@gmail.com \\ Universidad Cesar Vallejo, Chimbote \\ Perú \\ https://orcid.org/0000-0002-6936-1547 \\ Ana María Vargas-Castillo \\ vargascas5018@gmail.com \\ Universidad Cesar Vallejo, Chimbote \\ Perú \\ https://orcid.org/0000-0003-2045.8265 \\ Mery Marleny Martínez-lbarra \\ merymarlenymartinezibarra1@gmail.com \\ Universidad Cesar Vallejo, Chimbote \\ Perú \\ https://orcid.org/0000-0001-9714-063X \\ Ángela Beatriz Rodríguez-Montes \\ angelarodriguezmontes3@gmail.com \\ 431 \\ https://orcid.org/0000-0001-8404-1763
}

Recibido: 10 de marzo de 2021

Aprobado: 15 de junio de 2021 


\title{
RESUMEN
}

En estas circunstancias de la pandemia que se vive, el desempeño docente aparece inextricablemente entretejido al manejo y uso adecuado de las herramientas tecnológicas colaborativas y la virtualidad; pues, es la vía posible con la que se le está dando continuidad al proceso educativo. Considerando esto, se desarrolló la investigación tendiente a analizar el desempeño digital que tienen los docentes de Nuevo Chimbote en el contexto de la emergencia sanitaria por COVID-19. El estudio se desarrolló desde un enfoque metodológico cuantitativo, con un diseño no experimental, de campo, descriptivo y transversal. La muestra poblacional estuvo conformada por 1246 estudiantes, escogidos mediante la técnica muestral por cuota. Los docentes, en estas circunstancias de la emergencia sanitaria, han mostrado una actuación y desempeño acorde a las exigencias que generan procesos pedagógicos y de aprendizaje mediados por las TIC, es decir haciendo uso de la tecnología, la virtualidad y de herramientas digitales colaborativas.

Descriptores: Docente; desempeño; tecnologías. (Palabras tomadas del Tesauro UNESCO).

\begin{abstract}
In these circumstances of the current pandemic, teaching performance appears inextricably interwoven with the proper management and use of collaborative technological tools and virtuality; then, it is the possible way with which the educational process is being continued. Considering this, research was developed to analyze the digital performance of Nuevo Chimbote teachers in the context of the health emergency due to COVID-19. The study was developed from a quantitative methodological approach, with a non-experimental, field, descriptive and crosssectional design. The population sample consisted of 1,246 students, chosen using the quota sampling technique. The teachers, in these circumstances of the health emergency, have shown an action and performance of processes according to the demands that generate pedagogical and learning mediated by ICT, that is, making use of technology, virtuality and collaborative digital tools.
\end{abstract}

Descriptors: Teacher; performance; technologies. (Words taken from the UNESCO Thesaurus).

\section{INTRODUCCIÓN}


La pandemia provocada por la enfermedad de Covid19, es un problema sanitario que ha desencadenado, por un lado, en la cuarentena o prohibición del desplazamiento de las personas que posiblemente se encuentren infectados o que han estado expuestos a un potencial contagio; por otro lado, el distanciamiento social entendido como la restricción de la interacción entre las personas teniendo cierta distancia física a fin de prevenir el contacto directo entre ellas (Sánchez \& De la Fuente, 2020).

Cabe puntualizar, que estas medidas estratégicas asumidas para mitigar el virus y frenar el avance pandémico, han ocasionado múltiples cambios en todos ámbitos de la vida humana y generado una gran crisis a nivel mundial. Muestra de ello es que la Comisión Económica para América Latina y el Caribe (CEPAL) y la Oficina Regional de Educación para América Latina y el Caribe de la Organización de las Naciones Unidas para la Educación, la Ciencia y la Cultura (OREALC/UNESCO), reportan que, a partir de marzo de 2020, más de 190 países se vieron en la obligación de suspender las actividades presenciales de las instituciones educativas, de manera total o parcial, afectándose con ello a más de 1.200 millones de educandos de todos los niveles de enseñanza, lo que representa el $90 \%$ de la población estudiantil global (CEPAL \& OREALC/UNESCO, 2020).

Esta emergencia educativa causada por la interrupción de la actividad formativa presencial que se ha dado en gran parte de los países del mundo, se puede describir como una de las principales necesidades que se entrelaza a dicha crisis sanitaria y que es menester atender. En tal sentido, la mayoría de los gobiernos y sus sistemas educativos han asumido la responsabilidad de afrontar esta nueva realidad, pasando de una enseñanza presencial a una a distancia con uso de la tecnología y, como expresan Fardoun, Collazos \& Yousef (2020), "están trabajando fuertemente en intentar sostener los procesos de aprendizaje aplicando enfoques basados en la virtualización en algunas de sus actividades" (p.8).

Según el documento "La educación en tiempo de coronavirus: Los sistemas educativos de América Latina y el Caribe ante el Covid19" del Banco Interamericano de Desarrollo (BID), todas las acciones emprendidas para la continuidad de los servicios educativos durante el cierre de las escuelas e instituciones educativas, 


\section{CIENCIAMATRIA \\ Revista Interdisciplinaria de Humanidades, Educación, Ciencia y Tecnología \\ Año VII. Vol. VII. N¹3. Julio - Diciembre. 2021 \\ Hecho el depósito de ley: pp201602FA4721 \\ ISSN-L: 2542-3029; ISSN: 2610-802X \\ Universidad Nacional Experimental Francisco de Miranda (UNEFM). Santa Ana de Coro. Venezuela}

Silvia Lucía Galabay-Cajas; María Isabel Álvarez-Lozano

procuran entregar contenidos alineados al currículo escolar, acompañar el proceso de aprendizaje y mantener el vínculo estudiante-docente y familia-escuela (Banco Interamericano de Desarrollo [BID], 2020).

Es por ello que la enseñanza a distancia virtualizada, definida por Esposito \& Marsollier (2020) desde lo inscrito por Bonilla (2016), como "la educación a través del ciberespacio, posible mediante la conexión y uso de internet, no necesita de un tiempo y espacio específicos, y, permite establecer un nuevo escenario de comunicación entre docentes y estudiantes" (p.2); está siendo implementada en un lapso y velocidad sin precedentes, caracterizándose por contar con una combinación de medios y soluciones de primera generación (materiales impresos, radio y televisión) y de segunda generación (plataformas virtuales, sistemas de gestión de aprendizajes) para entregar contenido y mantener un nivel de interacción educativa óptima (BID, 2020). Pese a ello, muchos países de América Latina y el Caribe para el momento no tenían una estrategia de educación remota fortalecida y menos para atender una emergencia sanitaria, no obstante, han hecho esfuerzos ajustados con base en sus capacidades previas. Tal es el caso de Uruguay que ha sacado provecho de la infraestructura tecnológica que ha desarrollado desde el 2006 a través del Plan Ceibal y la democratización del acceso a la tecnología. En la actualidad es el único país de la región que tiene una plataforma integrada para la administración de los aprendizajes de los estudiantes, mediante el plan "Ceibal en casa", que le ha permitió pasar de las aulas de clase presencial a las virtuales en forma casi inmediata y, así, asegurar la continuidad del vínculo entre estudiantes y docentes, a pesar de los desafíos para llegar a las poblaciones más vulnerables, cuya conectividad a internet es limitada (Ripani \& Muñoz, 2020).

Por otro lado, países como Costa Rica, El Salvador, Guatemala, Honduras, Jamaica, Bahamas, Barbados, Trinidad y Tobago, Colombia y Perú cuentan con portales, con recursos análogos y digitales, disponibles a través de los sitios web de los ministerios de educación. Ejemplo de ello, es Colombia, país que cuenta con un portal educativo denominado "Colombia Aprende" con más de 80.000 recursos digitales disponibles para docentes y estudiantes en una amplia variedad de temas educativos, pero, que 


\author{
CIENCIAMATRIA \\ Revista Interdisciplinaria de Humanidades, Educación, Ciencia y Tecnología \\ Año VII. Vol. VII. N¹3. Julio - Diciembre. 2021 \\ Hecho el depósito de ley: pp201602FA4721 \\ ISSN-L: 2542-3029; ISSN: 2610-802X \\ Universidad Nacional Experimental Francisco de Miranda (UNEFM). Santa Ana de Coro. Venezuela
}

están diseñados para uso de docentes como apoyo en el aula o como complemento para el trabajo en casa. Asimismo, un grupo significativo de países como Argentina, Barbados, Belice, Brasil, Chile, Colombia, Costa Rica, Ecuador, Guatemala, Guyana, Haití, Honduras, Jamaica, México, Panamá, Perú, República Dominicana, Suriname, Venezuela y Trinidad \& Tobago, han reimpulsado el uso de la prensa, radio y televisión, como estrategias tecnológicas de primera generación (BID, 2020).

Otros países fuera del contexto latinoamericano y caribeño, también, han utilizado una diversidad de recursos y medios, tanto de primera como de segunda generación, para llegar a toda la población educativa. Así, Francia cuenta con la plataforma "Mi clase en casa" con un aula virtual y contenido digital de educación básica, ejercicios y proyectos. Dicha plataforma se complementa con la antigua France TV Education, ahora denominada Lumni.

Por su parte, Israel desarrolla la modalidad "blended" que implica la combinación de la educación presencial con la virtualidad a través de plataformas de contenidos y de comunicación en línea para actividades asíncronas (Moodle y Google Classroom). De igual manera, China puso en marcha una "nube nacional de educación a distancia" con clases básicas que cubren 12 asignaturas. En el caso de Italia y España, estos desarrollaron páginas web con herramientas, material multimedia y orientaciones para la continuidad de gestión educativa. Asimismo, los ministerios de educación de dichos países, acordaron con las televisoras la modificación de su parrilla matutina, para que destinaran más espacio a la educación y la cultura, e hicieron convenios con editoriales para difundir públicamente por televisión los contenidos digitales que acompañaban a los textos (BID, 2020).

Ahora bien, en el contexto peruano se estableció el aislamiento social obligatorio para reducir la cantidad de personas infectadas por el virus, según decreto de urgencia $\mathrm{N}$. $\stackrel{0}{-}$ 026-2020, a partir del 16 de marzo del 2020 y por espacio de 15 días, pero, se ha extendido hasta la fecha (Presidencia de la Republica de Perú, 2020). Es de saber que, en el marco de este decreto de aislamiento social, a nivel educativo y mediante resolución viceministerial $N^{\circ}$ 088-2020, se implementó la estrategia "Aprendo en Casa”, y, con ella, el conjunto de disposiciones que orientan el trabajo de los docentes 
para asegurar, en los niveles de educación inicial, primaria y secundaria, el desarrollo del servicio educativo a distancia usando diversos canales de comunicación, durante el estado de emergencia sanitaria por Covid19 (MINEDU, 2020)

Cabe destacar que según reporte del diario El Peruano de fecha 04 de junio de 2020, esta estrategia educativa "Aprendo en Casa" diseñada por el Minedu, ha sido elogiada por el diario norteamericano The Washington Post y las agencias internacionales de comunicación Deutsche Welle y Al Jazzera; además, de ser reconocida por su valor e incluida en la lista de buenas prácticas en la difusión de la cultura y las artes en América Latina, por parte de la Organización de las Naciones Unidas para la Educación, la Ciencia y la Cultura (Unesco).

A pesar de ello, el desarrollo de esta estrategia educativa "Aprendo en Casa" no ha sido fácil, pues, los docentes peruanos, los cuales no estaban preparados, se han visto obligados a auto capacitarse y rediseñar su trabajo. La tarea docente se ha visto recargada ya que el trabajo incluye la contextualización de las actividades de aprendizaje, retroalimentación a los estudiantes, revisión y devolución de las tareas, atención socioemocional a estudiantes, padres de familia y grupos organizados, usando WhatsApp, Messenger, Zoom u otras herramientas digitales. Tratando, a su vez, de superar los grandes desafíos como la accesibilidad a internet y el propio uso de herramientas tecnológicas (Wehrle, 2020 \& Estrada, 2020).

Lo anterior, ha implicado asumir, como expresan Esposito \& Marsollier (2020), el reto de mantener la vitalidad de la educación a través de la virtualidad. Es decir, promoviendo la interconexión de unos con otros a través de las redes sociales y/o herramientas colaborativas, para crear ecologías y dar forma a las comunidades de aprendizaje que conlleven a la producción y reproducción de conocimientos (Siemens, 2004 \& Giesbrecht, 2007 citados por Gutiérrez, 2012).

Asimismo, este desafío ha exigido y exige del docente la movilización de sus capacidades profesionales, su disposición personal y su responsabilidad social para articular relaciones significativas que impacten la formación del estudiante. En otras palabras, ha despertado un compromiso personal en el docente, que lo ha llevado a desempeñarse de tal manera que suscite en los estudiantes aprendizajes, actitudes, 
motivaciones, competencias y habilidades complejas, que les permitan participar en la sociedad y convertirse en aprendices autónomos a lo largo de la vida (ORELAC/UNESCO, 2012 en Escribano, 2018).

Como se observa claramente, en esta coyuntura de la pandemia que se vive, el desempeño del docente aparece inextricablemente entretejido al manejo y uso adecuado de las herramientas tecnológicas colaborativas y la virtualidad. Pues, es la vía posible con la que se le está dando continuidad al proceso educativo; considerando que, en esta era digital, la forma como trabajan y funcionan las personas para aprender, se altera cuando se usan nuevas herramientas tecnológicas y se cambian los ambientes de aprendizaje que permiten la interconexión en la realidad (Siemens, 2004 \& Giesbrecht, 2007 citado por Gutiérrez, 2012).

En este contexto, se hace necesario presentar algunos datos producto de otras investigaciones desarrolladas en este sentido. Cabe decir, estas investigaciones no solamente aluden al contexto de la emergencia sanitaria, en vista de que son muy pocos los estudios que se pueden encontrar en la web hasta ahora.

Primero, Picón, González \& Paredes (2020), con su estudio “Desempeño y formación docente en competencias digitales en clases no presenciales durante la pandemia COVID-19", realizado en 7 escuelas de la ciudad de Piribebuy del Paraguay, expresan que, en la emergencia sanitaria, el rol de los docentes ha sido indispensable para desplegar la experiencia de aprendizaje a distancia y mediada por las TIC o la infraestructura tecnológica provista para ello; puesto que, el docente sigue siendo fundamental para gestionar el aprendizaje de los estudiantes. Sin embargo, es primordial facilitar procesos de acompañamiento en el aprendizaje profesional de los docentes respecto a sus competencias referidas al uso de las tecnologías para producir un aprendizaje más efectivo.

Segundo, Tejedor, Cervi, Tusa \& Parola (2020), con su investigación "Educación en tiempos de pandemia: reflexiones de alumnos y profesores sobre la enseñanza virtual universitaria en España, Italia y Ecuador" realizada con estudiantes y docentes de tres universidad de los países mencionados, observan una valoración negativa sobre el paso a la virtualidad de la Educación Superior, destacándose que este cambio ha 


\section{CIENCIAMATRIA \\ Revista Interdisciplinaria de Humanidades, Educación, Ciencia y Tecnología \\ Año VII. Vol. VII. N¹3. Julio - Diciembre. 2021 \\ Hecho el depósito de ley: pp201602FA4721 \\ ISSN-L: 2542-3029; ISSN: 2610-802X \\ Universidad Nacional Experimental Francisco de Miranda (UNEFM). Santa Ana de Coro. Venezuela}

Silvia Lucía Galabay-Cajas; María Isabel Álvarez-Lozano

contribuido a impactar negativamente en la visión que el estudiantado posee de sus docentes en el escenario virtual. Tercero, Revilla \& Palacios (2020), con su estudio que busco evaluar el desempeño de los docentes en tiempos de pandemia desde la perspectiva de los estudiantes, realizado una universidad pública de Lima, concluyen que los estudiantes sitúan el desempeño del docente en un nivel preponderantemente medio, debido a que existe un déficit en el desarrollo de competencias tecnológicas por parte de los docentes que se ha hecho evidente durante el confinamiento. Cuarto, el estudio realizado por Moreno (2020), sobre la innovación educativa en tiempos de pandemia, destaca que una de las lecciones aprendidas en este contexto refieren a que la migración hacia ambientes virtuales de forma inesperada y abrupta por el estado de emergencia causa incertidumbre y angustia en docentes y estudiantes, debido a la pérdida de control de lo conocido. Es por ello, que el docente debe cambiar la metodología tradicional de enseñanza, ya que, la virtualidad exige mucho más trabajo y acompañamiento del docente para que realmente se evite la deserción de estudiantes y se logre la continuidad educativa para el alcance de los objetivos de aprendizaje.

Quinto, con respecto al desempeño digital de los docentes de educación inicial, se tiene que Moran \& Poma (2019), a razón de los resultados de su investigación relacionada con el manejo de las TIC en docentes de las instituciones educativas de educación inicial, realizada en el distrito de Huancavelica del Perú, enuncian que los docentes muestran un nivel medio de manejo de las TIC en el proceso de enseñanzaaprendizaje de los niños del mencionado nivel educativo. Esto se muestra con el medianamente manejo de algunos aspectos teóricos referidos a la computadora, navegadores; el uso parcial de la computadora en clases, videos, algunos buscadores, programas, entre otros; y, la poca capacidad para elaborar sus propios recursos didácticos digitales.

Sexto y último, en relación a la educación secundaria, Huamani, Valencia \& Ramírez (2019), con su artículo sobre el Programa EDUSOFT y desempeño docente en las instituciones educativas públicas del nivel secundario del Perú, concluyen que el desempeño docente está influenciado por el uso de las TIC; además, el nivel de 


\section{CIENCIAMATRIA \\ Revista Interdisciplinaria de Humanidades, Educación, Ciencia y Tecnología \\ Año VII. Vol. VII. N¹3. Julio - Diciembre. 2021 \\ Hecho el depósito de ley: pp201602FA4721 \\ ISSN-L: 2542-3029; ISSN: 2610-802X \\ Universidad Nacional Experimental Francisco de Miranda (UNEFM). Santa Ana de Coro. Venezuela}

Silvia Lucía Galabay-Cajas; María Isabel Álvarez-Lozano

afectación está determinado por factores como: el entrenamiento del maestro en cuanto al uso de las herramientas informáticas y la forma de aplicación pedagógica de estas en las clases. En tal sentido, es menester considerar el tema de la capacitación tecnológica, tanto para docentes como estudiantes, si se aspira adaptación más rápida al uso de las TIC en el proceso educativo.

A razón de lo planteado, y como indica el MINEDU (2012), se precisa del docente acciones concretas que aseguren una buena práctica educativa empleando la tecnología, especialmente aquélla relacionada con la información y comunicación (TIC), de manera coherente con las actividades de enseñanza y aprendizaje que se desarrollan en estos tiempos de incertidumbre que se viven por la pandemia.

Pues las TIC, como instrumentos determinados por los avances de la informática y las telecomunicaciones para la producción, interacción, tratamiento y comunicación de la información (Cruz, Pozo, Aushay \& Árias, 2019), en el en el ámbito educativo, representan un elemento fundamental de la dimensión didáctica que se constituyen en medios que facilitan la formación. Al mismo tiempo que están cambiando las formas de interrelación educativa entre docentes y estudiantes, fortaleciendo la socialización y el aprendizaje de los estudiantes, a través de nuevos conocimientos tecnológicos que, a su vez, fomentan la creatividad, la participación, el trabajo colaborativo, el desarrollar sus habilidades y valores como la tolerancia, respeto, solidaridad. Aunque ello, como expresan Lorenzo, Lorenzo \& Lledó (2019), demande un desempeño del docente marcado por nuevas habilidades y competencias digitales para asumir distintos entornos de aprendizaje y trabajos signados por las tecnologías, sobre todo en situaciones adversas como las actuales.

En virtud de todo lo expuesto y considerando a Revilla \& Palacios (2020), cuando exponen que el docente es un elemento crucial para el logro de los aprendizajes, por tanto, fortalecer todos los aspectos de su desempeño se convierte en un aspecto clave en el contexto de pandemia, se desarrolla el presente estudio en torno a los docentes del distrito de Nuevo Chimbote y en el marco de la estrategia "Aprendo en casa", a partir de cuestionar ¿Cómo perciben los estudiantes el desempeño docente en relación al manejo de la tecnología, la virtualidad y las herramientas digitales durante 
la pandemia? De allí que, el objetivo de investigación se enmarca en analizar el desempeño digital que tienen los docentes de Nuevo Chimbote en el contexto de la emergencia sanitaria por COVID-19.

\section{METODOLOGÍA}

Considerando los argumentos de Palella \& Martins (2015), se declara que el estudio se desarrolló desde un enfoque metodológico cuantitativo, fundamentado en el positivismo. A razón de ello, el diseño corresponde con una investigación no experimental, transversal, de campo; pues, los datos e informaciones se recabaron sin manipulación de variable alguna, en un momento único y directamente de las instituciones del Distrito de Nuevo Chimbote como ámbito donde ocurren los hechos. Cabe resaltar, que el estudio se desarrolló a nivel descriptivo, ya que, se buscó analizar el desempeño en relación al manejo de la tecnología, la virtualidad y las herramientas digitales que tienen los docentes como hecho, tal cual como ocurre en el contexto de la emergencia sanitaria por COVID-19.

En cuanto a la muestra poblacional estuvo conformada por un total de 1246 estudiantes, escogidos mediante la técnica muestral por cuota, considerándose el criterio para dicha escogencia que fuesen estudiantes del Distrito de Nuevo Chimbote, de los niveles de Inicial, Primaria y Secundaria de la Educación Básica peruana (Palella \& Martins, 2015). Por consiguiente, las cuotas de muestra poblacional estuvieron constituidas por 490 estudiantes de Inicial, 573 de Primaria y 183 de Secundaria.

Se utilizó como técnica para recabar los datos, una encuesta dirigida a los estudiantes de los 3 niveles, la cual se desplego a través de un cuestionario formulado de manera online, de carácter anónimo y constituido por 10 preguntas para los estudiantes de inicial y 20 preguntas para los estudiantes de primaria y secundaria. Dicho cuestionario fue validado a través de la técnica de juicios de expertos; además, se estableció su confiabilidad mediante la aplicación de 3 pruebas piloto, una por cada nivel, para calcular el Coeficiente de Alfa de Cronbach. En tal sentido, este coeficiente revela que es un instrumento de muy alta confiabilidad, pues, arrojó un $r=0.923$. Para 


\section{CIENCIAMATRIA \\ Revista Interdisciplinaria de Humanidades, Educación, Ciencia y Tecnología \\ Año VII. Vol. VII. N¹3. Julio - Diciembre. 2021 \\ Hecho el depósito de ley: pp201602FA4721 \\ ISSN-L: 2542-3029; ISSN: 2610-802X \\ Universidad Nacional Experimental Francisco de Miranda (UNEFM). Santa Ana de Coro. Venezuela}

Silvia Lucía Galabay-Cajas; María Isabel Álvarez-Lozano

cerrar, el procesamiento y análisis de los datos se efectuó con base en la estadística descriptiva (Palella \& Martins, 2015).

\section{RESULTADOS Y DISCUSIÓN}

El objetivo principal de este trabajo fue analizar el desempeño que tienen los docentes de Nuevo Chimbote en relación al manejo de la tecnología, la virtualidad y las herramientas digitales desde la percepción de los estudiantes y en el marco de la estrategia educativa "Aprendo en Casa" implementada para abordar la crisis educativa a razón de la emergencia sanitaria por COVID-19. En tal sentido y en función de los resultados producto de la encuesta aplicada a la globalidad de 1246 estudiantes encuestados en los tres niveles de educación básica regular del distrito de Nuevo Chimbote, se tiene que el 91,65\% (equivalente a 1142 encuestados), perciben un buen desempeño del docente al desarrollar procesos pedagógicos y de aprendizaje mediados por las TIC, es decir haciendo uso de la tecnología, la virtualidad y de herramientas digitales colaborativas, en contraposición al 8,35\% (lo que es igual 104 encuestados) que observan un desempeño docente tipificado de regular a deficiente. Estos resultados serían consistentes con autores como: ORELAC/UNESCO (2012) en Escribano (2018); Wehrle (2020) \& Estrada (2020); Lorenzo, Lorenzo \& Lledó (2019), Moreno (2020); Siemens (2003) \& Giesbrecht (2007), en Gutiérrez (2012) y Esposito \& Marsollier (2020), los cuales han expresado que la pandemia ha desafiado al docente a superar grandes retos como la accesibilidad a internet, el propio uso de herramientas tecnológicas, lo que se traduce en incertidumbre, en angustia; y, a vigorizar sus capacidades, habilidades, competencias y, sobre todo, su disposición y compromiso personal para asumir distintos escenarios y desempeñarse de tal manera que han podido crear, junto con estudiantes y padres de familia, espacios y ecologías de aprendizaje para la construcción de conocimientos y saberes; a la vez que se ha avivado y fortalecido la educación a través de la virtualidad y el uso de las TIC.

En cuanto al desempeño de los docentes de educación inicial, cuota que representa el $39,32 \%$ de los estudiantes en la totalidad de la muestra poblacional estimada, se revela que el $93,06 \%$ de los encuestado señalan que los docentes muestran un buen 


\section{CIENCIAMATRIA \\ Revista Interdisciplinaria de Humanidades, Educación, Ciencia y Tecnología \\ Año VII. Vol. VII. N¹3. Julio - Diciembre. 2021 \\ Hecho el depósito de ley: pp201602FA4721 \\ ISSN-L: 2542-3029; ISSN: 2610-802X \\ Universidad Nacional Experimental Francisco de Miranda (UNEFM). Santa Ana de Coro. Venezuela}

Silvia Lucía Galabay-Cajas; María Isabel Álvarez-Lozano

desempeño, ya que han podido desplegar el proceso pedagógico virtualizado creando sus propios recursos digitales y haciendo uso de herramientas tecnológicas colaborativas asequibles para ellos, a diferencia del 6,94\% que expresan percibir a los docentes con un desempeño de regular a deficiente. Estos datos alusivos al desempeño de los docentes de educación inicial, discrepan con lo expuesto por Moran \& Poma (2019) en relación a que los docentes de educación inicial muestran un nivel medio de manejo de las TIC. Es decir, requieren ser fortalecidos en sus competencias digitales para un mejor desempeño en el proceso de enseñanza-aprendizaje de los niños de dicho nivel educativo, aunque haya que considerando que el estudio fue realizado antes de la pandemia.

Respecto al desempeño de los docentes de educación primaria, cuota que constituye el $45,98 \%$ de la generalidad de la muestra poblacional estudiada, se puede exponer que el $94,93 \%$ de los estudiantes encuestados manifiestan que los docentes tienen un buen desempeño, en disconformidad con el 5,07\% de los encuestados que precisan un desempeño de regular a deficiente. Estos resultados se pueden entretejer con lo referido por Picón, González \& Paredes (2020), en cuanto a que el papel de docente en la emergencia sanitaria ha sido imprescindible para desarrollar la experiencia de aprendizaje a distancia, mediada por la virtualidad y las TIC. Sin embargo, es esencial el proceso de acompañamiento para el fortalecimiento de competencias, concernientes a las tecnologías y la virtualización para producir aprendizajes más efectivos en los estudiantes.

En relación al desempeño de los docentes de educación secundaria, cuota que compone el $14,68 \%$ del conjunto de la muestra poblacional, se enuncia que el $77,60 \%$ de los estudiantes encuestados indican que los docentes tienen un desempeño que se puede puntualizar de bueno, resultados que discrepan con el $22,40 \%$ de los estudiantes que revelan percibir de regular a deficiente el desempeño de los docentes. Hallazgo que se confronta con lo expresado por Tejedor, Cervi, Tusa \& Parola (2020) y Revilla \& Palacios (2020), a razón de que observan una valoración negativa del estudiantado hacia los docentes en el escenario virtual, Esto, debido a que el paso de la educación presencial a la virtual, a causa del confinamiento, ha sido 
impactante y ha puesto en evidencia las deficiencias en cuanto a las competencias para el uso de la tecnología y las herramientas digitales colaborativas por parte de los docentes.

Para cerrar, cabe resaltar que los hallazgos encontrados son indicativos de que los docentes, en estas circunstancias de la emergencia sanitaria, han mostrado una actuación y desempeño acorde a las exigencias y como se ha estipulado en el Marco del Buen Desempeño Docente (MINEDU, 2012). Y, a pesar de las limitaciones que supone desenvolverse en este contexto de incertidumbre y angustia, han podido crear estrategias de acompañamiento y ambientes adecuados para el logro de los aprendizajes de manera remota por parte de los estudiantes. En otras palabras, han asumido los cambios y retos que representa pasar de una educación presencial a una remota basada en la virtual, así sea temporalmente.

\section{CONCLUSIONES}

Vivimos en un mundo de constantes cambios e incertidumbres, por tanto, se debe estar preparado para asumir situaciones coyunturales y adversas, como las vividas en estos tiempos de emergencia sanitaria por COVID-19. En tal sentido y dado la situación de confinamiento, las aulas se han constituido desde los hogares y los docentes se han tenido que apropiar de nuevos enfoques fundamentales para la enseñanza remota, lo cual, ha desencadenado que el proceso de aprendizaje y la educación en general, estén experimentando una metamorfosis influenciada por la tecnología, la virtualidad y el uso de herramientas digitales.

Lo planteado, aunado a que los resultados de investigación develan el buen desempeño que han tenido de los docentes de Nuevo Chimbote en cuanto al manejo de la tecnología, la virtualidad y las herramientas digitales como estrategia fundamental que se despliega con el programa "Aprendo en Casa", se puede enunciar que la actuación de los docentes, en estos tiempos de confinamiento, ha sido de mucha tenacidad, ahínco y firmeza. Se ha despertado en ellos, el poder de imaginar nuevas formas y la capacidad de emprender ante los cambios y escenarios imprevisible; pues, al docente le toco reinventarse para estar acorde a las 
circunstancias y triunfar ante el desafío de mantener la vitalidad de la educación a través de la tecnología y la virtualidad.

Finalmente, a partir de los hallazgos, se aúpa a que la sociedad en su conjunto reconozca la labor de los maestros, quienes han podido superar las barreras tecnológicas y de la comunicación, que se han suscitado con el abrupto paso de la educación presencial a la remota y evidenciarse como profesionales de vanguardia. Asimismo, se sugiere que el gobierno, nacional y/o local, realice el soporte de competencias digitales con capacitación docente, $\mathrm{Y}$, al propio docente, que siga autoformándose en cuanto al conocimiento digital; pues, solo de esta forma se lograr la excelencia en el desempeño como profesional.

\section{REFERENCIAS}

Banco Interamericano de Desarrollo [BID] (2020). La educación en tiempos del coronavirus. Los sistemas educativos de América Latina y el Caribe ante el Covid19 [Education in times of the coronavirus. The educational systems of Latin America and the Caribbean in the face of Covid19]. Recuperado de https://n9.cl/3oh5f

CEPAL \& OREALC/UNESCO (2020). La educación en tiempos de pandemia de Covid19 [Education in times of the Covid19 pandemic]. Recuperado de https://n9.cl/3oh5f

Cruz, M., Pozo, M., Aushay, H. \& Arias, A. (2019). Las Tecnologías de la Información y de la Comunicación (TIC) como forma investigativa interdisciplinaria con un enfoque intercultural para el proceso de formación estudiantil [Information and Communication Technologies (ICT) as an interdisciplinary investigative form with an intercultural approach for the student training process]. e-Ciencias de la Información. 9(1). DOI: https://doi.org/10.15517/eci.v1i1.33052

Escribano, E. (2018). El desempeño del docente como factor asociado a la calidad educativa en América Latina [Teacher performance as a factor associated with educational quality in Latin America]. Revista Educación. 42(2). DOI DOI http://dx.doi.org/10.15517/revedu.v42i2.27033 
Esposito, C. \& Marsollier, R. (2020). Virtualidad y educación en tiempos de COVID19. Un estudio empírico en Argentina [Virtuality and education in times of COVID-19. An empirical study in Argentina]. Educación y Humanismo. 22(39), 1-22. DOI: https://doi.org/10.17081/eduhum.22.39.4214

Estrada, P. (2020). El aprendizaje remoto enfrenta a otro reto: el profesorado no está preparado para la enseñanza en línea. Observatorio de Innovación Educativa [Remote learning faces another challenge: teachers are not ready for online teaching. Observatory of Educational Innovation]. Recuperado de https://n9.cl/96bvh

Fardoun, H., González, C., Collazos, C., \& Yousef, M. (2020). Estudio exploratorio en lberoamérica sobre procesos de enseñanza - aprendizaje y propuesta de evaluación en tiempos de pandemia [Exploratory study in Latin America on teaching-learning processes and evaluation proposal in times of pandemic]. Education in the Knowledge Society (EKS).21(9). DOI: https://doi.org/10.14201/eks.23537

Gutierrez, L. (2012). Conectivismo como teoría de aprendizaje: conceptos, ideas, y posibles limitaciones [Connectivism as a learning theory: concepts, ideas, and possible limitations]. Revista Educación y Tecnología. 1, 111-122. Recuperado de http://revistas.umce.cl/index.php/edytec/article/view/39

Huamani, T., Valencia, A. \& Ramírez, F. (2019). Programa EDUSOFT y desempeño docente en las instituciones educativas públicas del nivel secundario [EDUSOFT program and teaching performance in public educational institutions at the secondary level]. PURIQ. 1(02), 240-252. DOI https://doi.org/10.37073/puriq.1.02.37

Lorenzo, G., Lorenzo, A. \& Lledó, A. (2019). Las TIC en el contexto educativo a través de la producción científica en español [ICT in the educational context through scientific production in Spanish]. Revista General de Información y Documentación. 29(2), 287-307. DOI https://doi.org/10.5209/rgid.66969

Ministerio de Educación [MINED] (2020). Resolución Viceministerial № 088-2020 [Vice Ministerial Resolution No. 088-2020]. Recuperado de https://n9.cl/x7fey

Ministerio de Educación [MINED] (2012). Marco del buen desempeño docente para mejorar tu practica como maestro y guiar el aprendizaje de tus estudiantes [Framework of good teaching performance to improve your practice as a teacher and guide the learning of your students]. Recuperado de https://n9.cl/4g1e2 


\section{Silvia Lucía Galabay-Cajas; María Isabel Álvarez-Lozano}

Morán, M. \& Poma, I. (2019). Manejo de tecnologías de información y comunicación (TICS) en docentes de las instituciones educativas de educación inicial del distrito de Huancavelica. (tesis de postgrado) [Management of information and communication technologies (ICT) in teachers of educational institutions of initial education of the district of Huancavelica. (postgraduate thesis)]. Recuperado de https://repositorio.unh.edu.pe/handle/UNH/2522

Moreno, S. (2020). La innovación educativa en los tiempos del Coronavirus [Educational innovation in the times of the Coronavirus]. Salutem Scientia Spiritus. 6(1), 14-26. Recuperado de https://revistas.javerianacali.edu.co/index.php/salutemscientiaspiritus/article/vi $\underline{\mathrm{ew} / 22}$

Palella, S. \& Martins, F. (2015). Metodología de la Investigación Cuantitativa [Quantitative Research Methodology]. Caracas. FEDUPEL.

Picón, G., González, G. \& Paredes, J. (2020). Desempeño y formación docente en competencias digitales en clases no presenciales durante la pandemia COVID19 [Performance and teacher training in digital skills in non-face-to-face classes during the COVID-19 pandemic]. DOI: $\underline{10.1590 / \text { SciELOPreprints. } 778}$

Presidencia de la Republica de Perú (2020). Decreto de Urgencia № 026-2020 [Emergency Decree No. 026-2020]. Recuperado de https://n9.cl/3u7y

Revilla, J. \& Palacios, A. (2020). Ser docente en tiempos de pandemia por Covid-19: evaluación del desempeño docente en una universidad pública de Lima [Being a teacher in times of a Covid-19 pandemic: evaluation of teaching performance at a public university in Lima]. Ágora. 7(2), 58-62. Disponible en: https://www.revistaagora.com/index.php/cieUMA/article/view/117

Ripani, M. \& Muñoz, M. (2020). Plan Ceibal 2020: Desafíos de innovación educativa en Uruguay [Plan Ceibal 2020: Challenges of educational innovation in Uruguay]. Fundación Ceibal, Montevideo. Recuperado de https://digital.fundacionceibal.edu.uy

Sanchez, A. \& De la Fuente, V. (2020). COVID19: Cuarentena, aislamiento, distanciamiento social [Quarantine, isolation, social distancing]. Anales de Pediatria. 93(1), 73-74. Recuperado de https://n9.cl/4fngr 
Revista Interdisciplinaria de Humanidades, Educación, Ciencia y Tecnología

Año VII. Vol. VII. N¹3. Julio - Diciembre. 2021

Hecho el depósito de ley: pp201602FA4721

ISSN-L: 2542-3029; ISSN: 2610-802X

Universidad Nacional Experimental Francisco de Miranda (UNEFM). Santa Ana de Coro. Venezuela

Silvia Lucía Galabay-Cajas; María Isabel Álvarez-Lozano

Tejedor, S., Cervi, L., Tusa, F. \& Parola, A. (2020). Educación en tiempos de pandemia: reflexiones de alumnos y profesores sobre la enseñanza virtual universitaria en España, Italia y Ecuador [Education in times of pandemic: reflections of students and teachers on virtual university education in Spain, Italy and Ecuador]. Revista Latina de Comunicación Social. 78, 1-21. DOI https://www.doi.org/10.4185/RLCS-2020-1466

Wehrle, A. (2020). Educación en contextos de Covid19: requerimientos mínimos para una educación a distancia. Observatorio Educativo Ciudadano [Education in Covid19 contexts: minimum requirements for distance education. Citizen Educational Observatory]. Recuperado de https://n9.cl/gkdyl 